\title{
Stability comparison of two ring resonator structures for multiwavelength fiber lasers using highly doped Er-fibers
}

\author{
Rosa Ana Pérez-Herrera, M. Angeles Quintela, Montserrat Fernández-Vallejo, Antonio Quintela, \\ Manuel López-Amo Senior Member, IEEE Jose Miguel Lopez-Higuera, Senior Member, IEEE
}

\begin{abstract}
An experimental comparison of stability between two different fiber laser topologies is carried out. The lasers are based on ring resonators that include highly-doped Er-fibers. Both topologies use fiber Bragg grating reflectors in order to select the emission wavelengths. The experimental results confirms that the novel topology based on fiber optic circulators arranged in an hybrid serial-parallel configuration offers a better stability and higher optical signal to noise ratios (OSNR) than the simpler based on a parallel configuration.
\end{abstract}

Index Terms - Erbium-doped fiber (EDF), fiber Bragg grating (FBG), optical fiber ring laser, optical fiber amplifier, wavelength division multiplexing (WDM).

\section{INTRODUCTION}

$\mathrm{M}$ ULTIWAVELENGTH erbium doped fiber lasers (MEDFL) have attracted attention due to its potential applications in both sensing and telecommunication fields (wavelengthdivision multiplexing, fiber-optic sensors, optical spectroscopy, etc) [1]. Among the different types of EDFL, the ring laser is a very competitive structure to achieve simultaneously the requirements of many of these applications: narrow linewidth and high output optical power. Another important advantage is their large range wavelength tunability [2].

The selection of their operation wavelengths has been achieved by using different optical filtering devices like Mach-Zehnder filters [3], arrayed waveguide gratings (AWGs) [3], [4], or fiber Bragg gratings (FBGs) [5], [6]. In addition to this, a variety of methods have been employed to try to maximize the number of emission lines and to improve their stability [3], [7], [8], [9].

FBGs are also widely used as optical fiber sensors. They offer many advantages over electronic sensors on certain applications. Among fiber-optic sensors, FBGs have a

Manuscrited received August, 4 2008. This work was supported in part by the Spanish Government TEC2007-67987-C02, European COST-299

R. A. P-H, M. F-V, M. L-A. authors are with the Department of Electric and Electronic Engineering, Public University of Navarra, Pamplona, Spain. (corresponding author to provide rosa.perez@unavarra.es)

M. A. Q., A. Q. and J. M. L. authors are with the Photonics Engineering Group, University of Cantabria, ETSIIT Avda. Los Castros s/n, 39005 Spain growing importance given their simplicity, small size, flexible design and low losses. FBGs are usually employed as strain and/or temperature sensors, encoding the information of the measured parameter on their operation wavelength. Combining these two fields, EDFRL sources and FBG sensors, multiwavelength sources and multiplexing systems for fiber sensor networks can be obtained [5].

An important aspect to take into account of the MEDFL is its output power instability and mode competition [10]. These instabilities can degrade the performance characteristics of a sensor array based on a tunable ring laser interrogation scheme. The design parameters optimization of these lasers, such as erbium doped fiber (EDF) length and the coupling ratio can reduce these undesirable effects [10]. Moreover, an appropriate election of a ring laser configuration can improve considerably the characteristics of these lasers.

In this paper, a comparison between two different MEDFL topologies is carried out. The first topology was previously reported using another kind of Er-doped fibers [1] and the second one is a novel one based on the serial connection of the FBGs using optical circulators. Experimental results of the temperature and time stability of the two MEDFL are also presented.

Both studied structures show a good temporal stability for four wavelengths. As is reported in literature ring lasers with more than 2 [11] or 3 [12] wavelengths are instable. Our structures show also good time stability for 4 wavelengths without cooling the EDF to $77 \mathrm{~K}$ with liquid nitrogen as reported in [13].

\section{EXPERIMENTAL SET UPS}

\section{A. Two Wavelength Lasers}

The first experimental setups of proposed MEDFRLs are shown in Fig. 1. The wavelength selection will be carried out by means of FBGs. The operation wavelengths of the FBGs have been located in the flat region of the erbium gain profile in order to achieve good equalization between channels. A highly doped Er-fiber (Er-30, with absorption of $10.8 \mathrm{~dB} / \mathrm{m}$ at $\lambda=978 \mathrm{~nm}$ by Liekki) was incorporated, acting as the active medium. The length of this highly doped erbium fiber was 7 $\mathrm{m}$. Because of this, the fiber length needed for the cavity was 
shorter than in other cases [1].

One of the major problems in multiwavelength ring lasers is correctly adjusting the cavity losses on each wavelength in order to achieve oscillation of the system in all the desired channels. The oscillation threshold power for each wavelength is different due to the nonflat shape of the erbium fiber gain profile; as a consequence, individual loss control for each wavelength is required. In order to resolve this problem, a variable attenuator (VA) has been connected to each FBG. All the free terminations on both systems have been immersed in refractive-index-matching gel to avoid undesired reflections.

The first configuration (Fig. 1(a)) was composed by the highly doped Er-fiber, an optical isolator, a polarization controller (PC), a 1480/1550nm wavelength division multiplexers (WDM), two fiber Bragg gratings (FBG) and a $3 \mathrm{~dB}$ coupler to incorporate the two FBGs into the laser cavity. To extract $2 \%$ of the laser output power from the ring, a $98 \%$ coupler is used. The pump laser wavelength utilized in this configuration was $1480 \mathrm{~nm}$. The optical isolator ensures unidirectional oscillation in the ring cavity. The polarization controller provides an intracavity mechanism to compensate for the slight polarization dependent gain. The Bragg wavelengths of the FBGs determine the lasing wavelengths of the EDFRL. The FBGs have the Bragg wavelengths of $1543.05 \mathrm{nn}$ and $1550.52 \mathrm{~nm}$ and the full-width at half maximum (FWHM) of $0.842 \mathrm{~nm}$ and $0.61 \mathrm{~nm}$, respectively. Optical power inside the ring is thus divided into two branches of approximately equal power. Each of these branches is composed of a FBG and a VA, used to regulate the losses of the corresponding wavelength in order to achieve oscillation in all the channels.

The second fiber laser analyzed experimentally is a new configuration where optical circulators are used to insert the FBGs reflected signals into the ring (Fig. 1b). Because of this, the utilization of isolators to ensure the unidirectional oscillation in this configuration was not necessary because the circulators accomplish this function.

In this novel configuration two circulators were used to direct the signal inside the ring, ensuring unidirectional operation and therefore avoiding the spatial hole-burning effect. Each branch is composed of a FBG, an optical circulator and a VA. The advantages of this topology in comparison with the typical in-line filtering topology, where the FBG and VA of each channel are placed consecutively and inserted into the ring through an unique circulator, is the ease of the individual control of each channel. The variations of the first channels have not effect on the following channels. Nevertheless, the main drawback is the difficulty to incorporate a new channel because this involves adding a new optical circulator.

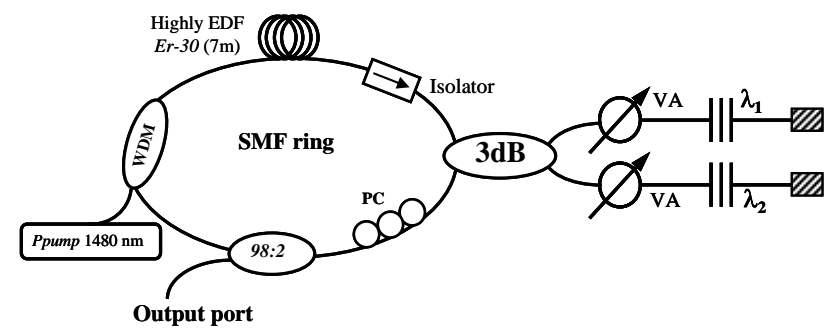

(a)

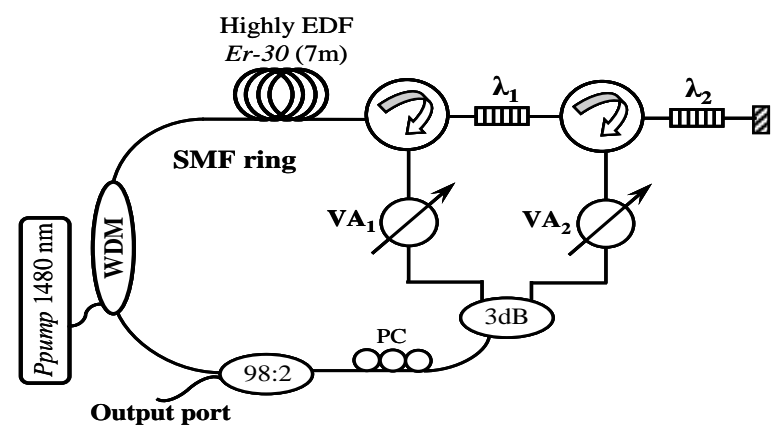

(b)

Fig. 1 (a). Experimental setup of the EDFRL based on the parallel topology with a $2 \times 2$ coupler. (b). Experimental setup of the new EDFRL configuration with circulators.

\section{B. Four Wavelength Lasers}

Now we are going to check the performance of these structures for 4 wavelengths. We have eliminated the isolator in order to compare the configurations with previously reported in [1], that was also checked for four wavelengths and for a pump laser operating at $980 \mathrm{~nm}$.

These experimental setups comprised also a standard single-mode fiber (SMF) ring in which the same highly doped Er-fiber (Er-30, with absorption of $10.8 \mathrm{~dB} / \mathrm{m}$ at $\lambda=978 \mathrm{~nm}$ by Liekki) was incorporated, acting as the active medium. Once again, the amplification is obtained by using 7 meters of this highly doped erbium fiber. Additionally, the 1480-nm pump source was replaced for a 980-nm pump source and a wavelength division multiplexer (WDM) were used to form the ring resonator. In this case, gratings of 1539.92, 1545.15, 1550.031, and $1555.079 \mathrm{~nm}$ have been used, each one showing $0.27 \mathrm{~nm}, 0.27 \mathrm{~nm}, 0.26 \mathrm{~nm}$ and $0.29 \mathrm{~nm}$ at $-3 \mathrm{~dB}$ bandwidth in that order and $96.2 \%, 99.9 \%, 92.4 \%$ and $99.9 \%$ reflectivity correspondingly. To characterize the laser stability, we measured the output power by means of an optical spectrum analyzer (OSA).

The experimental setup for this new EDFRL is shown in Fig. 2(a). On this occasion, the $3 \mathrm{~dB}$ coupler was substituted for a $2 \mathrm{X} 4$ coupler to incorporate four FBGs into the laser cavity, creating a star or parallel topology. Optical power inside the ring is thus divided into four branches of approximately equal power. A coupler is used to extract part of the EDFA output power from the ring to the optical spectrum analyzer (OSA). 


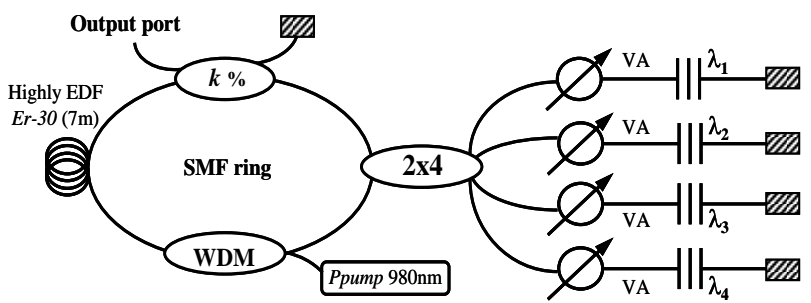

(a)

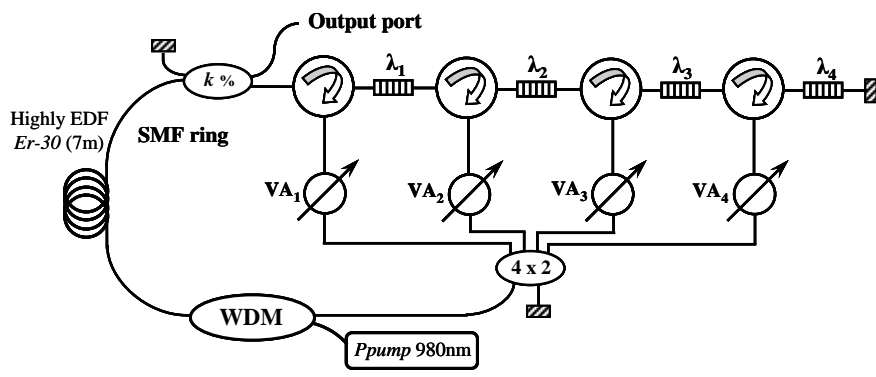

(b)

Fig 2. (a) Experimental setup for the parallel topology with couplers. (b) Experimental setup for the new topology with circulators.

Fig. 2(b) shows the next studied and analyzed configuration. The power was extracted via different outcoupling ratios in order to study the dependence on these two parameters. As well as an output power stability analyses with the temperature and time for one of these FBGs was carried out. In order to improve the OSNR (measurement of the ratio of signal power to noise power in an optical channel) of both structures, the results for a new location of the output port was also studied and analyzed. This new location of the output port can be seen in the Figure 3(a) and Figure 3(b). In the second configuration, due to this new location for the output port, the coupler inside the ring was unnecessary.

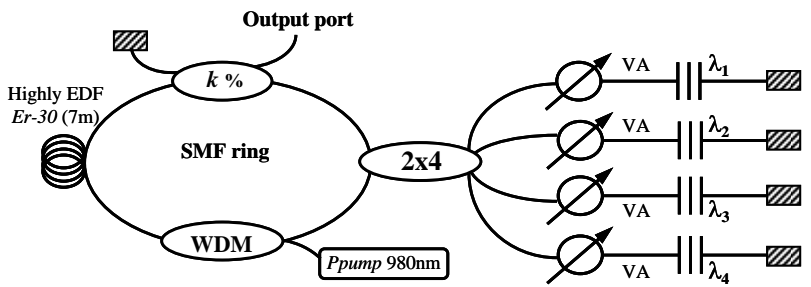

(a)

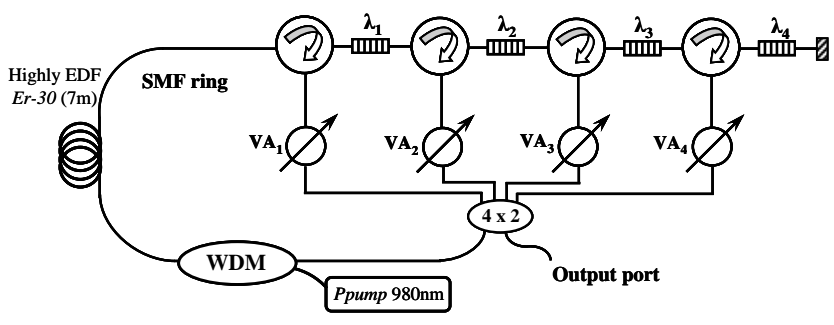

(b)

Fig. 3. (a) New location of the output port in the parallel topology with $2 \mathrm{X} 4$ coupler. (b) New location of the output port in the serial topology with circulators

\section{RESULTS}

The power and the wavelength of the laser output can suffer some changes with the time. So, in this first part the output power and the signal wavelength stabilities are both experimentally measured on both ring fiber laser structures with two lasing wavelengths. The instability is defined as the output power and wavelength variations $(\mathrm{dB}$ and $\mathrm{nm}$ ) for a given interval of time (hours) and a specific confidence level (CL), that is the probability value associated with a confidence interval, given as a percentage[15]. The confidence interval (CI) is the estimated range of values where the parameter of interest is included. We have tested each laser structure for eleven different pump powers (from $55 \mathrm{~mW}$ to $250 \mathrm{~mW}$ ), maintaining each pump power during 6 hours. The measured data have been stored each 15 seconds, using four different CL of $99 \%, 90 \%, 80 \%$ and $75 \%$, respectively. Depending on the CL, the confidence interval of the output power $\left(\mathrm{CI}_{\mathrm{p}}\right)$ or the signal wavelength $\left(\mathrm{CI}_{\mathrm{w}}\right)$ is defined. As a consequence, the instability of the output power and the signal wavelength is calculated within the corresponding $\mathrm{CI}$. The $\mathrm{CI}_{\mathrm{x}}$, where $\mathrm{x}$ is $\mathrm{p}$ or $\mathrm{w}$ for the output power or the signal wavelength, respectively, is defined as

$$
\mathrm{CI}_{\mathrm{x}}=\left(\mathrm{Y}_{\mathrm{cx}}-\mathrm{FU}_{\mathrm{x}}, \mathrm{Y}_{\mathrm{cx}}+\mathrm{FU}_{\mathrm{x}}\right)
$$

where $Y_{c x}$ is the average of the output power or signal wavelength, $\mathrm{U}_{\mathrm{x}}$ is the standard deviation and $\mathrm{F}$ is the uncertainty factor that shows the CL. For example, for $\mathrm{F}=1$; $\mathrm{CL}=66 \%$, for $\mathrm{F}=2 ; \mathrm{CL}=95 \%$ and for $\mathrm{F}=3 ; \mathrm{CL}=99 \%$.

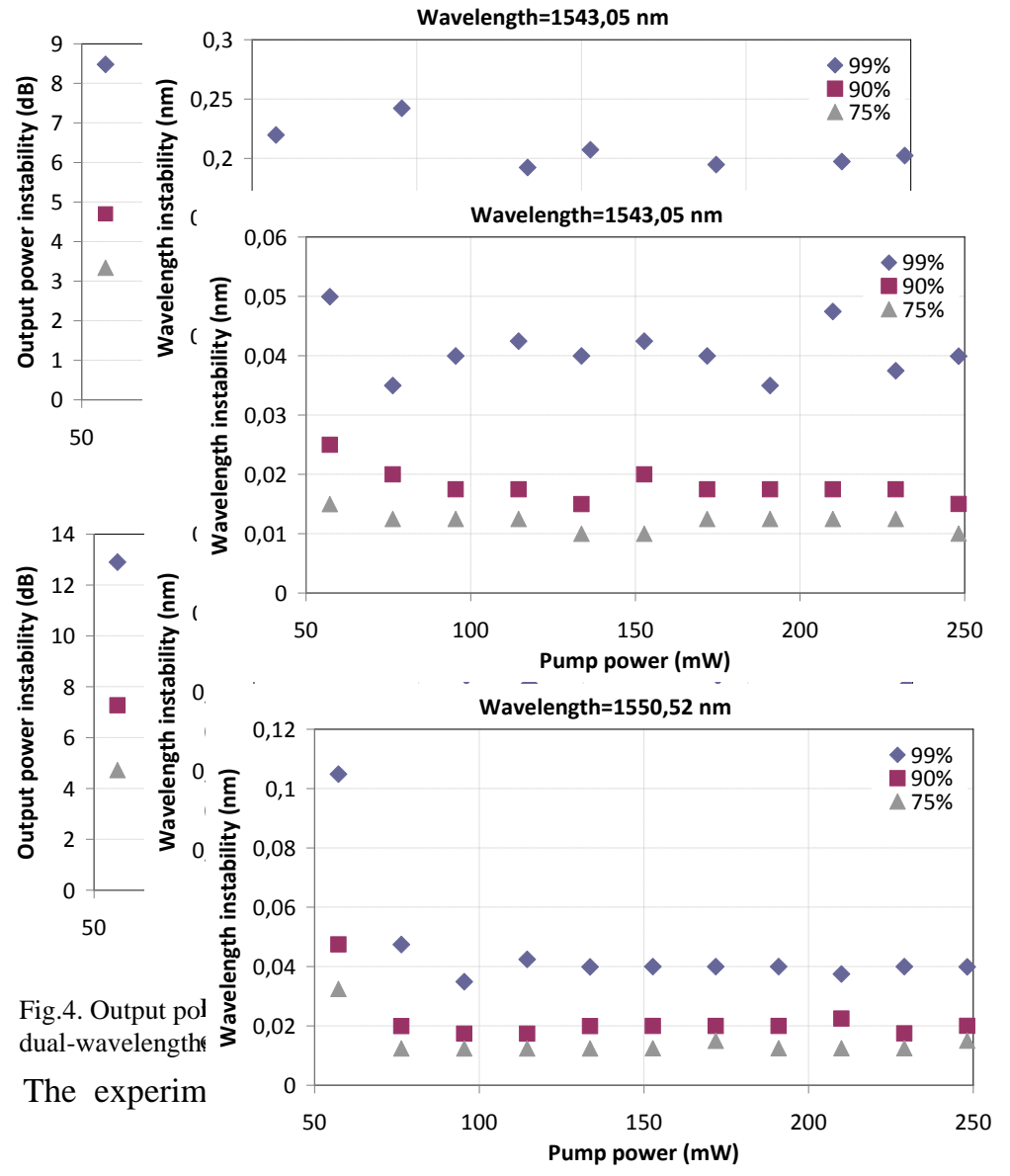

Fig.7. Wavelength instability as a function of the pump power to the dual-wavelengths EDFRL with circulators. Authorized licensed use limited to: UNIV DE NAVARRA. Downloaded on April 28, 2009 at 08:05 from IEEE Xplore. Restrictions apply. 
wavelengths EDFRL based on a parallel topology as a function of the pump power for different CL are shown in Fig. 4. The output power instability depends on the pump power for both signal wavelengths. High pump powers are required for a better stability and with a further pump power increase remains almost unchanged. For $\mathrm{CL}=90 \%$ and $\lambda_{1}=1543.05 \mathrm{~nm}$ the worst measured output power instability value is $4.7 \mathrm{~dB}$ for a pump power of $57 \mathrm{~mW}$ and the best value is $2.4 \mathrm{~dB}$ for a pump power of $210 \mathrm{~mW}$. And for $\mathrm{CL}=90 \%$ and $\lambda_{2}=1550.52$ $\mathrm{nm}$ the worst measured output power instability value is 7.3 $\mathrm{dB}$ for a pump power of $57 \mathrm{~mW}$ and the best value is $2.4 \mathrm{~dB}$ for a pump power of $210 \mathrm{~mW}$.

The experimental results of the output power instability of dual-wavelengths EDFRLs based on circulators as a function of the pump power for different CL are shown in Fig. 5. The output power instability as a function of the pump power presents a similar behavior than the other fiber laser. As can be shown in this figure the power instability is reduced considerably with this new laser configuration. For $\mathrm{CL}=90 \%$ and $\lambda_{1}=1543.05 \mathrm{~nm}$ the worst measured output power instability value is $1.6 \mathrm{~dB}$ for a pump power of $57 \mathrm{~mW}$ and the best value is $0.4 \mathrm{~dB}$ for a pump power of $172 \mathrm{~mW}$. And for $\mathrm{CL}=90 \%$ and $\lambda_{2}=1550.52 \mathrm{~nm}$ the worst measured output power instability value is $2.3 \mathrm{~dB}$ for a pump power of $57 \mathrm{~mW}$ and the best value is $0.17 \mathrm{~dB}$ for a pump power of $172 \mathrm{~mW}$. This can be explained by the fact that in practice these circulators present a better optical isolation that the isolator, and as a consequence, the power in the backward direction is lower with this novel configuration.
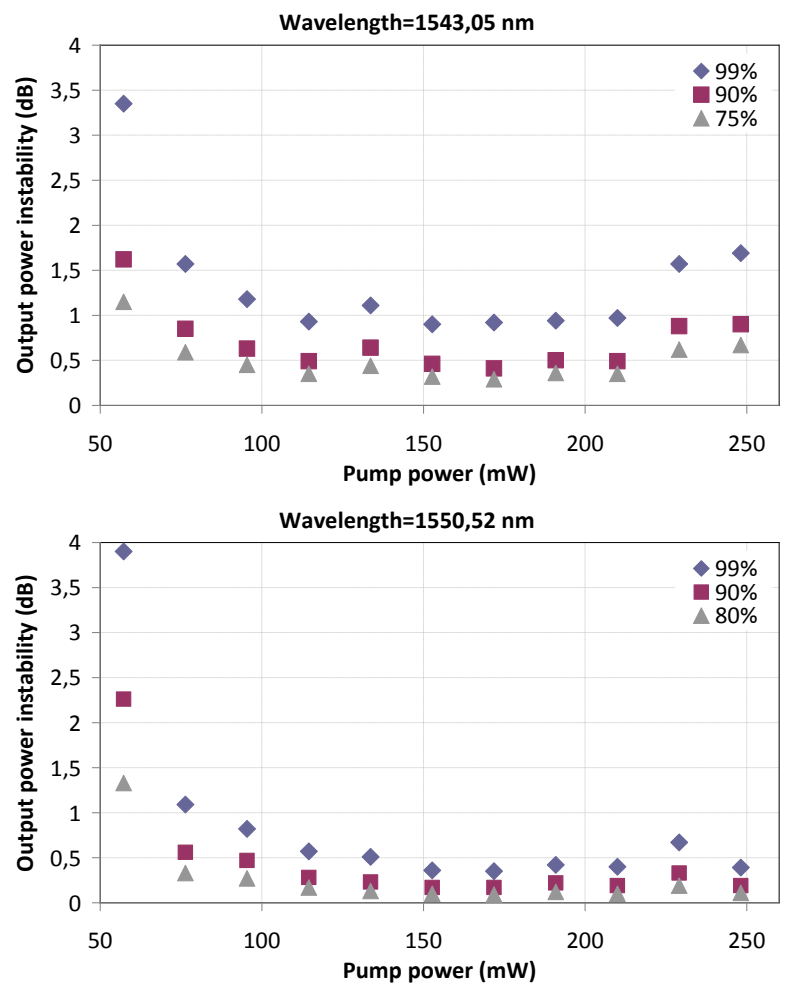

Fig.5.Output power instability as a function of the pump power to the dual-wavelengths EDFRL with circulators.
The signal wavelength instability was also measured for different value of CL. The experimental results are shown in Fig. 6 and 7. If these experimental results with both laser configurations are compared, it can be deduced that the wavelength stability is less dependent on the pump power with the EDFRL based on circulators. Moreover the wavelength instability is reduced one order of magnitude with the EDFRL based on circulators. The reason of this behavior is the same that with the power instability. A summary of the best values of power and wavelength stability for both laser configurations and a CL of $90 \%$ are shown in the table I.

TABLE I

MINIMUM VALUES OF THE OUTPUT POWER AND WAVELENGTH INSTABILITY FOR BOTH MEDFRLS.

\begin{tabular}{|c|c|c|c|c|}
\cline { 2 - 5 } \multicolumn{1}{c|}{} & \multicolumn{2}{c|}{ Power instability (dB) } & \multicolumn{2}{c|}{ Wavelength instability $(\mathrm{nm})$} \\
\cline { 2 - 5 } & $1543.05 \mathrm{~nm}$ & $1550.52 \mathrm{~nm}$ & $1543.05 \mathrm{~nm}$ & $1550.52 \mathrm{~nm}$ \\
\hline $\begin{array}{c}\text { MEDFRL } \\
\text { with } \\
\text { couplers }\end{array}$ & 2.4 & 2.4 & 0.062 & 0.11 \\
\hline $\begin{array}{c}\text { MEDFRL } \\
\text { with } \\
\text { circulators }\end{array}$ & 0.41 & 0.17 & 0.015 & 0.017 \\
\hline
\end{tabular}

Once the two-wavelength emission laser was studied and analyzed, two more FBGs were inserted in this set ups and some variations were done in order to simplify it; such as the elimination of the polarization mode control because of not having significant impact on the stability [14]. As a first step, we have studied the relation between the coupling ratio $(K)$ of the power-extraction coupler and the OSNR as well as the output power of the four resulting channels. Optimization of this parameter is of the utmost importance; for that reason this study have done for several coupling ratios for each configuration before system characterization. This can be seen in Fig 8 and Fig 9.

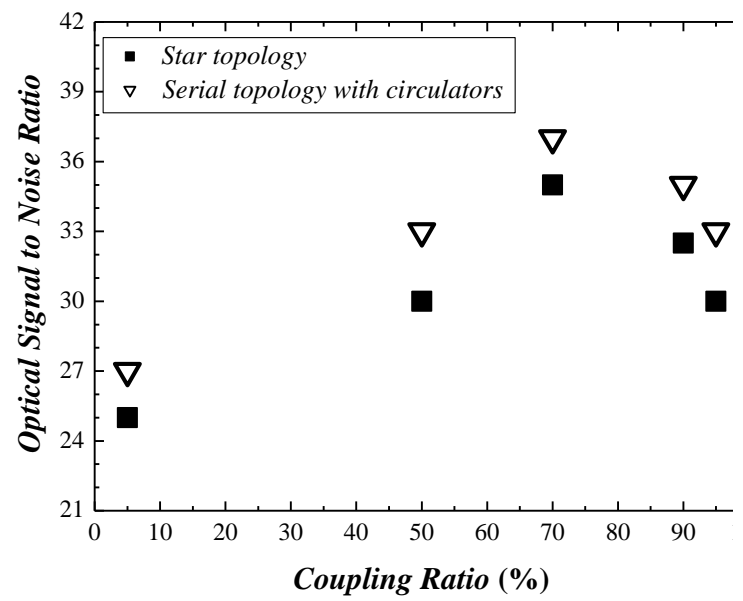

Fig. 8. Variations of the optical signal to noise ratio for both configurations. 


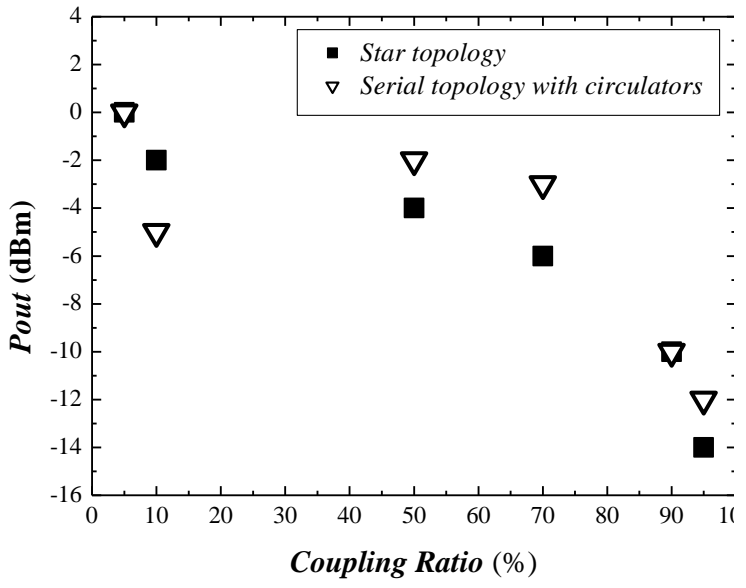

Fig. 9. Variations of the output power level for both topologies.

A compromise between OSNR and output power has to be considered. In this case, optimum coupling ratio for both structures were about $K=70 \%$ and this was the out-coupling ratio used in our experimental studies. The output spectrums of this fiber lasers for a $220 \mathrm{~mW}$ pump power are shown in Fig. 10(a) and Fig. 10(b) for the couplers and circulators configuration correspondingly. The power of each of the four output channels is around $-6 \mathrm{dBm}$ and $-3 \mathrm{dBm}$ respectively, and the power differences between them are about 0.5 and 0.7 $\mathrm{dB}$ in that order. For every channel, the signal power is more than $35 \mathrm{~dB}$ higher than the ASE noise floor.
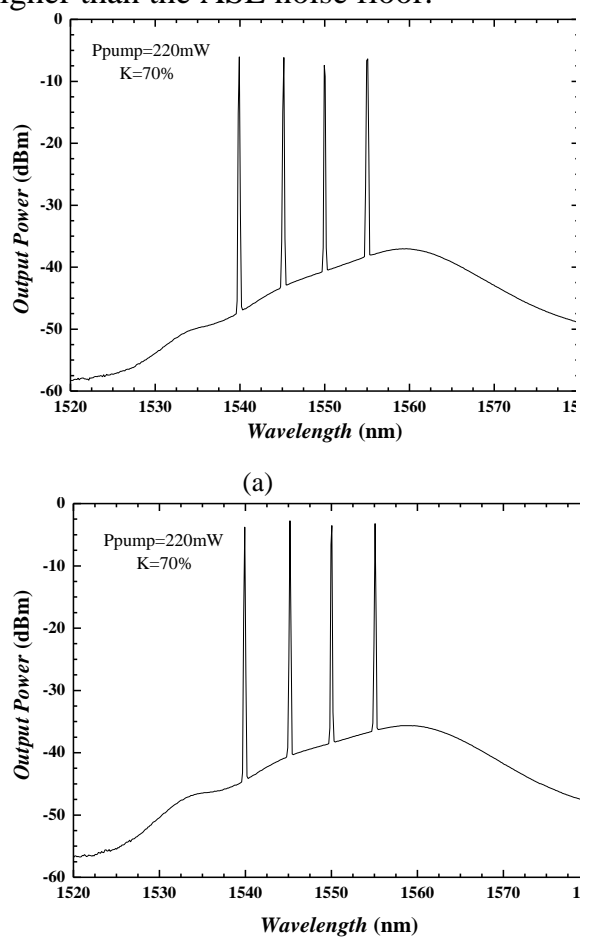

(b)

Fig. 10. (a) Output spectrum for the couplers configuration. (b) Output spectrum for the circulators configuration.

The power stability of these structures versus temperature variations was also analyzed. Therefore, one of the FBGs was placed inside a climatic chamber and temperature cycles from $25^{\circ} \mathrm{C}$ to $100^{\circ} \mathrm{C}$ were performed in order to study its behavior. Four tests were carried out for each structure to obtain an averaged measurement for all these results. For the first configuration, the power variation was around $7 \mathrm{~dB}$ for the FBG of $1545.15 \mathrm{~nm}$. For the second one, this variation was $8.7 \mathrm{~dB}$.

In order to eliminate the ASE noise floor related to the EDFA, the output port location was changed. Instead of locating at the output of the coupler inside the ring, the output port was connected to the coupler and circulator structure as it can be shown in Fig 3(a) and Figure 3(b). Fig 11(a) and Fig 11(b) represent the output spectrum for these two new configurations.
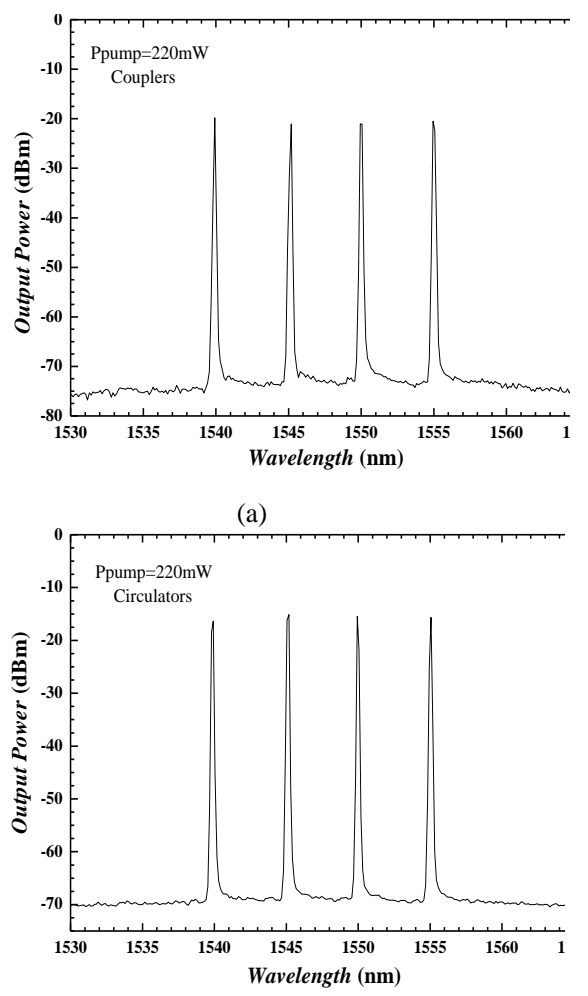

(b)

Fig. 11. (a) Output spectrum for the star configuration with ASE suppression (b) Output spectrum for the circulators configuration with ASE suppression

The OSNR was improved for both structures due to the ASE suppression. With preciseness, this value was increased from about $35 \mathrm{~dB}$ to $52 \mathrm{~dB}$ for the first configuration and from around $37 \mathrm{~dB}$ to $55 \mathrm{~dB}$ for the second one. These OSNR are $20 \mathrm{~dB}$ higher than previously reported in [1] Nevertheless, the output power of the four channels was decreased from around $-6 \mathrm{dBm}$ to $-20 \mathrm{dBm}$ for the first configuration and from -3 $\mathrm{dBm}$ to $-15 \mathrm{dBm}$ for the second one. Consequently, a compromise between OSNR and output power has to be considered. In this case, the power stability depending on the time was also analyzed. For that cause, each output power channel was evaluated every 2 minutes with a length of one hour at a room temperature. For the first configuration, the output power instability was about $7.45 \mathrm{~dB}$ for the output channels. For the second structure, the output power 
instability was around $2.78 \mathrm{~dB}$ for the same output channels, so we can conclude that the stability at room temperature using the structure with circulators is much better than using the structure with couplers.

\section{CONCLUSION}

An experimental comparison of stability between two different fiber laser topologies has been carried out. The lasers are based on ring resonators that include highly-doped Er-fibers. Both topologies use fiber Bragg grating reflectors in order to select the emission wavelengths. From the experimental results, it is found that the EDFRL based on a serial configuration of FBGs using circulators presents a better stability of both output power and signal wavelength than the EDFRL based on a parallel configuration. Another important aspect of this new configuration is that the output power stability is almost unchanged with high pump powers with the laser configuration with circulators. It is also proven that the signal wavelength variation is considerably reduced and it is practically invariable with the pump power.

On the other hand, the dependence between the coupling ratio and the optical signal to noise ratio and the output power for the channels was analyzed, as well as the high dependence between the temperature and the power stability for the channels, which was also experimentally pointed out; showing that the new configuration based on circulators has also a better performance. Finally, the results for both topologies when the ASE noise is suppressed had been shown and analyzed, viewing how the OSNR had been increased remarkably.

\section{ACKNOWLEDGMENT}

The authors want to thank Liekkis' fibers for having supplied the erbium doped fiber used in the experiments.

\section{REFERENCES}

[1] L. Talaverano, S. Abad, S. Jarabo, and M. López-Amo, "Multiwavelength Fiber Laser Sources with Bragg-Grating Sensor Multiplexing Capability," Journal of Lightwave Technology, vol. 19, n. 4, (2001), pp. 553-558.

[2] M. A. Quintela, C. Quintela, M. Lomer, F. J. Madruga, O. M. Conde, J. M. Lopez-Higuera, "Comparison between a symmetric bidirectionalpumping and a unidirectional-pumping configurations in an erbium fiber ring laser," EWOFS 2007 Third European Workshop on Optical Fibre Sensors, (2007), pp. 661920.

[3] A. Bellemare, "Continuous-wave silica-based erbium-doped fibre lasers," Progress in Quantum Electronics 27, (2003), pp. 211-266

[4] T. Miyazaki, N. Edagawa, S. Yamamoto, and S. Akiba, "A multiwavelength fiber ring-laser employing a pair of silica-based arrayed-waveguide-gratings," IEEE Photonics Technology Letters, vol. 9, n. 7, (1997), pp. 910-912.

[5] A. D. Kersey and W. W. Morey, "Multi-element Bragg-grating based Fiber-laser Strain Sensor," Electronics Letters, vol. 29, n. 11, (1993), pp. 964-966.

[6] E. Achaerandio, S. Jarabo, S. Abad, and M. López-Amo, "New WDM Amplified Network for Optical Sensor Multiplexing," IEEE Photonics Technology Letters, vol. 11, n. 12, (1999), pp. 1644-1646.

[7] H. L. An, X. Z. Lin, E. Y. B. Pun, and H. D. Liu, "Multi-wavelength operation of an erbium-doped fiber ring laser using a dual-pass MachZehnder comb filter," Optics Communications, vol. 169, n. 1-6, (1999), pp. $159-165$.
[8] X. Liu, X. Yang, F. Lu, J. Ng, X. Zhou, and Chao Lu, "Stable and uniform dual-wavelength erbium-doped fiber laser based on fiber Bragg wgratings and photonic crystal fiber," Optics Express, vol. 13, n. 1, (2005), pp. 142-147.

[9] S. Yamashita and K. Hotate, "Multiwavelength erbium-doped fiber laser using intracavity etalon and cooled by liquid nitrogen," Electronics Letters, vol. 32, n. 14, (1996), pp. 1298-1299.

[10] A. Zhang, H. Liu, M. S. Demokan, H. Y. Tam, "Stable and broad bandwidth multiwavelength fiber ring laser incorporating a highly nonlinear photonic crystal fiber," IEEE Photonics Technology Letters, vol. 17, n 12, (2005), pp. 2535-2537.

[11] Chien-Hung Yeh, Fu-Yuan Shih, Chang-Tai Chen, Chien-Nan Lee, "Stabilized dual-wavelength erbium-doped dual-ring fiber laser" Optics Express, Vol. 15, n. 21 (2007), pp. 13844-13848

[12] Z. Chun-Liu, Y. Xiufeng, L. Chao, N. Jun Hong, G. Xin, P. Roy Chaudhuri, D. Xinyong, "Switchable multi-wavelength erbium-doped fiber lasers by using cascaded fiber Bragg gratings written in high birefringence fiber," Optics Communications, vol. 230, n. 4-6, (2004), pp. 313-317.

[13] H.L. An, X.Z. Lin, E.Y.B. Pun, H.D. Liu, "Multi-wavelength operation of an erbium-doped fiber ring laser using a dual-pass Mach-Zehnder comb filter," Optics Communications, vol. 169, n. 1-6, (1999), pp.159165.

[14] A. Gusarov, F. Liegeois, "Experimental study of a tunable fiber ring laser stability," Optics Communications, vol. 234, n. 1-6, (2004), pp. 391-397.

[15] http://www.stats.gla.ac.uk/steps/glossary/confidence intervals.html

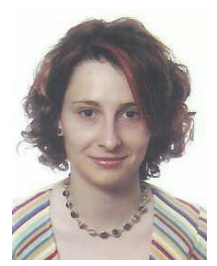

Rosa Ana Perez-Herrera was born in Cantabria, Spain, in February 1979. She received the telecommunications engineer degree from the University of Cantabria, Spain in 2004. In 2005 she obtained a scholarship from the Science and Technology Spanish Ministry and she joined the optical fiber sensor group at the Department of electrical and electronic engineering of the Public University of Navarre (Pamplona, Spain). She is currently working toward the Ph.D. degree in the "Communications" Doctoral program. Her research interests are in Raman amplifiers, erbium-doped amplifiers, fiberoptic sensors and multiplexing architectures.

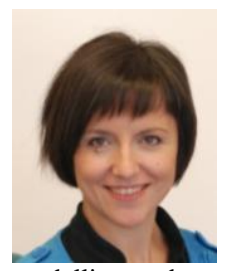

M. Angeles Quintela was born in Santander, Spain, in 1974. Se received both his Telecommunication Technical Engineering degree and his Telecommunication Engineering degree at the University of Cantabria. In 1998, she joined the University of Cantabria as lecturer. Her teaching topics include electronic components and optical communications. In 2005 she got his Ph.D. degree. Her primary research concern contains computer modelling and experimental characterisation of the fibre lasers. She has coauthored more than 70 papers presented in conferences and scientific journals

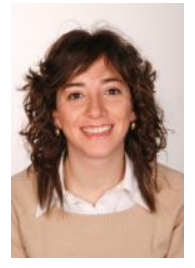

Montserrat Fernández Vallejo was born in Navarra, Spain, in February 1983. She received the telecommunications engineer degree from the Public University of Navarra, Spain in 2008. She is currently working toward the Ph.D. degree in the "Communications" Doctoral program. Her research interests are in Raman amplifiers, erbium-doped amplifiers, fiber-optic sensors and multiplexing architectures.

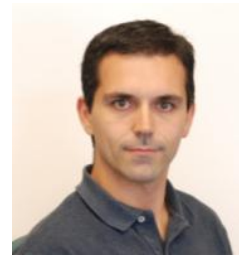

Antonio Quintela was born in Santander, Spain, in 1975. He received both his Telecommunication Technical Engineering degree and his Telecommunication Engineering degree at the University of Cantabria. In 1998 he joined the Photonics Engineering Group where, in 2006, he got his $\mathrm{Ph} . \mathrm{D}$. degree. His primary research concern is the writing of Fiber Bragg Grating (FBG) and fabrication of transducers based in FBG. He has co-authored more than 75 papers presented in conferences and scientific journals 


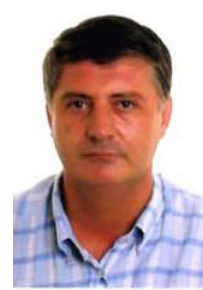

Manuel Lopez-Amo (M'91, SM '98) was born in Madrid, Spain, in 1960. He received the telecommunications engineer degree and Ph.D. degrees from the Universidad Politécnica de Madrid, Spain in 1985 and 1989, respectively. From 1985 to 1989 , he was a Lecturer in Optical Communications and Electronics at the Photonic Technology Department of the Universidad Politécnica de Madrid. In January 1990, he became an associate professor at the Photonic Technology Department of the

Universidad Politécnica de Madrid.

In 1996, he moved to Universidad Publica de Navarra (Pamplona, Spain) where he became Full Professor in the Electrical and Electronical Engineering department and is currently the head of the optical communications group of this department. He has been Chairman of the Optoelectronic Committee of Spain. He has been leader of more than 20 research projects and he has coauthored more than 100 works in international refereed journals and conferences related with fiber-optic networks, optical amplifiers, fiber-optic sensors, and integrated optics. He is a member of the technical committees of the International Conference on fiber optic sensors (OFS), the European Workshop on optical fibre sensors (EWOFS), and European cost 299 action, among others.

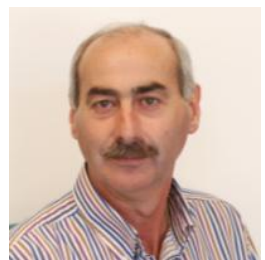

Jose Miguel López-Higuera was born in February 1954, in the village of Ramales de la Victoria, Cantabria (Spain). He obtained his Telecommunication Technical Engineering degree in the Universidad Laboral de Alcalá de Henares, and his Telecommunication Engineering degree in the Universidad Politécnica de Madrid (UPM). He achieved his $\mathrm{PhD}$ degree in Telecommunication Engineering, with an extraordinary award, in the UPM. He founded and is the head of the Photonics Engineering Group of the TEISA Department in the University of Cantabria. Presently, he works in the development of Photonics Instrumentation, Photonic/optical fiber sensor systems for civil engineering, electrical power, environmental and smart structures and for optical diagnostics for a wide set of sector applications. He has directed more that fifty $R \& D$ projects and has written or co-written more than four hundred publications in the form of books, chapters of books, papers and conferences, both national and international, and obtained 10 industrial publications (patents). His is the editor and co-author of several books. Among then can be mentioned: i) "Optical Sensors", UC, 1998; ii) the "Handbook of Photonic Sensing Technology“",Wiley and Sons, 2002 and iii) he is the co-editor of the book "Engineering a High-Tech Business: Entrepreneurial experiences and Insights", published by SPIE-Press,USA, 2008. Professor López-Higuera is senior member of the IEEE and member of the IEE, SPIE and OSA. 